Check for updates

Cite this: RSC Adv., 2017, 7, 50176

Received 11th September 2017 Accepted 16th October 2017

DOI: 10.1039/c7ra10111b

rsc.li/rsc-advances

\section{One-pot preparation of poly(styrene-co- divinylbenzene)/silver nanoparticles composite microspheres with tunable porosity and their catalytic degradation of methylene blue in aqueous solution}

\author{
Xiaohuan Ji, ${ }^{a}$ Franziska Griesing, ${ }^{\mathrm{b}}$ Ruijia Yan, ${ }^{a}$ Bin Sun, (D) *a Werner Pauer, ${ }^{* b}$ \\ Meifang Zhu, ${ }^{\text {*a }}$ Yushan Sun ${ }^{\text {ac }}$ and Hans-Ulrich Moritz ${ }^{b}$
}

\begin{abstract}
A series of poly(styrene-co-divinylbenzene)/silver nanoparticle (P(St-DVB)/AgNP) composite microspheres with tunable porosity were synthesized by seed swelling polymerization in one pot using linear polystyrene seeds as templates and nanosized organic soluble AgNPs as the silver source. The polymerization of styrene and divinylbenzene took place inside the seed particles and AgNPs were dispersed directly into the mixture of styrene and divinylbenzene during the polymerization. The morphology properties of P(St-DVB)/AgNPs microspheres were characterized by optical microscope, scanning electron microscope (SEM) and nitrogen adsorption/desorption. The influencing parameters on the morphology of the microspheres, including feed ratio of styrene (monomer) and divinylbenzene (crosslinker), addictive amount of AgNPs and the variety of the porogens were systematically investigated. The porosity of the composite microspheres could be tuned from nonporous to mesoporous and to macroporous by control over these parameters. Meanwhile, AgNPs loading dispersity of the composite microspheres was characterized by transmission electron microscope (TEM), energy-dispersive X-ray spectroscopy (EDS), SEM and thermogravimetric analysis (TGA). TEM, EDS and SEM results demonstrated that the AgNPs had been loaded both inside and on the surface of the microspheres. The sizes of the loaded AgNPs were influenced by the addictive amount of AgNPs and the kinds of porogens used in the polymerization. Moreover, compared to the P(St-DVB) microspheres, the Ag-loading composite microspheres synthesized in this paper exhibited excellent catalytic activity and reusability in the degradation of methylene blue in the presence of $\mathrm{NaBH}_{4}$.
\end{abstract}

\section{Introduction}

Over the last decades silver nanoparticles (AgNPs) have attracted great research interest in various fields, such as antibacterial agents, ${ }^{1,2}$ surface-enhanced Raman scattering, ${ }^{3}$ biosensing ${ }^{4}$ and catalysis. $^{5}$ As an efficient catalyst, AgNPs are widely used in degradation of organic dye pollutants, ${ }^{6}$ 4-nitrophenol, ${ }^{7}$ oxidation of ethane ${ }^{8}$ and splitting water into $\mathrm{H}_{2}$ and $\mathrm{O}_{2} \cdot{ }^{9}$ However, single AgNPs used as catalytic materials very easily become aggregated due to their high surface energy and that usually leads to

\footnotetext{
${ }^{a}$ State Key Laboratory for Modification of Chemical Fibers and Polymer Materials, College of Materials Science and Engineering, Donghua University, Shanghai, 201620, P. R. China. E-mail: zmf@dhu.edu.cn; sunbin@dhu.edu.cn; Fax: +86-2167792855; Tel: +86-21-67792849

${ }^{b}$ Institute for Technical and Macromolecular Chemistry, University of Hamburg, Bundesstraße 45, Hamburg, 20146, Germany. E-mail: pauer@chemie.uni-hamburg. de; Fax: +49-40-42838-6008; Tel: +49-40-42838-6007/3164

${ }^{c}$ State Key Laboratory of Biobased Fiber Manufacturing Technology, China Textile Academy, Beijing, 100025, P. R. China
}

a remarkable reduction in their catalytic activities. ${ }^{10}$ In addition, an efficient catalyst should be easily recycled and reused.

To solve these problems, organic and inorganic support materials are usually used to load AgNPs, for example carbonbased materials (carbon nanotubes, ${ }^{\mathbf{1 1}}$ carbon-based nanofibers, ${ }^{12}$ and graphene oxide $\left.{ }^{13}\right)$, polymer materials, ${ }^{\mathbf{1 4}, 15}$ metal oxide, ${ }^{16} \mathrm{TiO}_{2},{ }^{17}$ and mesoporous silica, ${ }^{18}$ etc. Among those supporting substrates, porous polymeric microspheres with high specific surface area, tunable pore morphology, strong adsorption capacity, excellent physicochemical stability and stable mechanical properties are becoming a superior choice. ${ }^{19-21}$ For example, silver nanoparticles have been immobilized in mesoporous cross-linked polyacrylic acid to catalyze the reduction coupling of nitrobenzenes and alcohols. ${ }^{22}$ Kim et al. ${ }^{23}$ used a fine colloidal silver to deposit silver nanoparticles on functional porous poly(ethylene glycol dimethacrylate) microspheres with controllable surface area and surface functionality. Zha et $a .^{24}$ prepared multi-porous sulfonated polystyrene/chitosan/Ag microspheres and compared their catalytic efficiency of reduction methylene blue with solid 
structured composite particles. The results indicated the porous structured spheres could efficiently adsorb chromium ions and the catalytic degradation rate was significantly faster than that of solid structured composite particles. So the porous structures of the surface and interior make porous polymer microspheres totally different from the conventional polymer microspheres without any pores. Especially in the application field of catalysis, the porous structure plays an important role in determining the efficiency of the reactant adsorption performance and the contact area between the catalysts and the reactants.

Traditionally, the approaches to develop well-defined and crosslinked microspheres with pore structures include suspension, precipitation and dispersion, membrane/ microchannel emulsification and microfluidic polymerizations. ${ }^{25-29}$ Among the mentioned methods, seed suspension polymerization based on polystyrene seeds as templates has received more considerable attention as controllable particle size, facile fabrication, tunable pore structures, and easy functionalization..$^{30,31}$ For instance, silver nanoparticles loaded on porous poly(styrene-co-divinylbenzene) microspheres with controllable interconnected structures were achieved by a suspension polymerization based on water-in-oil-in-water emulsion template using monooleate as the oil phase. ${ }^{32}$ In a further example of silver nanoparticles loaded monodisperse and multihollow poly(styrene-co-sodium 4-vinylbenzenesulfonate) microspheres, the composite microspheres were prepared using seed swelling polymerization by reducing $\left[\mathrm{Ag}\left(\mathrm{NH}_{3}\right)_{2}\right]^{+}$ions with polyvinylpyrrolidone. ${ }^{33}$ Like the silver nanoparticles and polymer composite microspheres mentioned above, silver nanoparticles incorporated in porous compounds are usually achieved by wet impregnation of porous materials with silver precursors in solution phase and subsequent reduction to metallic silver, which is a multi-step and timeconsuming process. Therefore, there is still a critical need to develop a more facile and feasible method to prepare porous polystyrene/silver nanoparticles composites.

Herein, the synthesis of porous P(St-DVB)/AgNPs composite microspheres in a one-pot seed swelling polymerization method is described. Polystyrene seeds and organic soluble AgNPs powders are prepared previously. The monomer with crosslinker, silver nanoparticles, initiator and porogens are mixed together in the second swelling step, so the reaction happens inside the swollen PS seeds. The AgNPs used in this experiment are well dispersed in the monomer, crosslinker and porogens, so the AgNPs can be easily absorbed into the PS seeds as well as dispersed inside the final microspheres. The porosity of the $\mathrm{P}(\mathrm{St}-\mathrm{DVB}) / \mathrm{AgNPs}$ composite microspheres was regulated successfully from nonporous, mesoporous to macroporous by changing the monomer and crosslinker ratio, the concentration of AgNPs added during the polymerization and the variety of porogens. Catalytic activity of the P(St-DVB)/AgNPs composite microspheres with different AgNPs loading and porosity were evaluated through degradation of methylene blue in the aqueous solution with $\mathrm{NaBH}_{4}$. High reusability was demonstrated without significant decrease in the catalytic performance after running 10 catalytic cycles.

\section{Experimental}

\subsection{Materials}

For the synthesis of silver nanoparticles, silver nitrate $\left(\mathrm{AgNO}_{3}\right.$, $>99.9 \%$, Shanghai Chemical Corporation, China), oleic acid (90.0\%, Aldrich), n-butylamine (99.5\%, Sigma-Aldrich) and Lascorbic acid (99.0\%, Sigma-Aldrich) were used as received. For the preparation of polystyrene seed particles, styrene $(\geq 99.0 \%$, Aldrich) was distilled under reduced pressure to remove the inhibitors prior to the use, polyvinylpyrrolidone (PVP-K30, $M_{\mathrm{n}}=$ $40 \mathrm{~kg} \mathrm{~mol}^{-1}, \mathrm{BASF}$ ), ethanol (99.8\%, VWR chemicals), Aliquat 336 (Aldrich) and azobisisobutyronitrile (AIBN, $\geq 98.0 \%$, SigmaAldrich) were applied as received. For the polymerization of the composite microspheres, sodium dodecyl sulfate (SDS, $\geq 95.0 \%$, Carl Roth, Germany), dibutyl phthalate (DBP, 99.0\%, Merck chemicals), divinylbenzene (DVB, 99.0\%, Merck chemicals), dibenzoyl peroxide (DBPO, 99.8\%, Sigma-Aldrich), toluene (99.8\%, Acros Organics), $n$-heptane (99.0\%, Grüssing), $n$-hexadecane ( $\geq 99.0 \%$, Merck chemicals), poly(vinyl alcohol) (PVA, Mowiol 18-88, Aldrich) and tetrahydrofuran (THF, 99.9\%, SigmaAldrich) were used without further purification. Methylene blue (MB) and sodium borohydride $\left(\mathrm{NaBH}_{4}, \geq 98.0 \%\right)$ used for catalytic experiment were purchased from Sigma-Aldrich and used as received. Deionized water was used for all the experiments.

\subsection{Preparation of organic soluble silver nanoparticles}

Organic soluble AgNPs were synthesized referring to our previous work. ${ }^{34}$ Oleic acid $(0.27 \mathrm{~mol}, 90 \mathrm{~mL})$ and $n$-butylamine $(1.50 \mathrm{~mol}, 150 \mathrm{~mL})$ were dispersed in $400 \mathrm{~mL}$ deionized water in a three-necked flask. Then $\mathrm{AgNO}_{3}$ aqueous solution (0.08 M, 50 $\mathrm{mL}$ ) was added into the flask with vigorous stirring. The reaction temperature was heated to $50{ }^{\circ} \mathrm{C}$ using an oil bath and the rate of continuous stirring was $400 \mathrm{rpm}$. After $20 \mathrm{~min}$, the solution had become transparent and an aqueous solution of ascorbic acid $(0.08 \mathrm{M}, 100 \mathrm{~mL})$ was quickly added to the transparent solution. A colloidal AgNPs solution was obtained after the reaction had taken place for $2 \mathrm{~h}$. The AgNPs colloid was cooled to room temperature. Ethanol was added into the AgNPs colloid, the sediment was washed with acetone 3 times. After drying in the vacuum oven at the temperature of $25^{\circ} \mathrm{C}$ for $24 \mathrm{~h}$, AgNPs powders were obtained.

\subsection{Preparation of polystyrene seed particles}

Polystyrene (PS) seed particles were prepared by dispersion polymerization. ${ }^{35}$ Solution 1 was a mixture made of ethanol (120 g), polyvinylpyrrolidone (PVP, $3.0 \mathrm{~g}$ ) and Aliquat 336 (1.2 g) in a three-necked $250 \mathrm{~mL}$ double walled glass reactor, which were placed on a shaking plate. The solution was purged with argon for $15 \mathrm{~min}$, before the temperature was increased to $70{ }^{\circ} \mathrm{C}$ by the means of a recirculating heater. Solution 2 was prepared in a separate flask, in which styrene (30 g) and AIBN (0.6 g) were mixed and purged with argon for 10 minutes, before it was added to the preheated solution 1 . The reactor was sealed and the polymerization took place at a shaking rate of $150 \mathrm{rpm}$ for $24 \mathrm{~h}$ and was stopped by cooling to room temperature. Seed latex particles were purified by repeated washing and centrifugation 
using ethanol, hot water, and ethanol in sequence. Lastly, the particles were dried under vacuum.

\subsection{Preparation of porous $\mathrm{P}(\mathrm{St}-\mathrm{DVB}) / \mathrm{AgNPs}$ composite microspheres}

The porous $\mathrm{P}(\mathrm{St}-\mathrm{DVB}) / \mathrm{AgNPs}$ composite microspheres were synthesized using two-step seed swelling polymerization. During the first step of swelling, the polystyrene seed particles $(0.2 \mathrm{~g})$ were dispersed in $1.8 \mathrm{~g}$ of $0.25 \mathrm{wt} \%$ aqueous sodium dodecyl sulfate (SDS) solution using sonification and were added to a sonificated emulsion of dibutyl phthalate (DBP, $0.5 \mathrm{~g}$ ) in $50 \mathrm{~g}$ $0.25 \mathrm{wt} \%$ SDS solution. The mixture was stirred at $400 \mathrm{rpm}$ for $5 \mathrm{~h}$ at room temperature. In the second step swelling, a separately prepared emulsion containing styrene and DVB with different weight ratios, AgNPs, porogen ( $0.8 \mathrm{~g}$ ), dibenzoylperoxide (DBPO, $0.05 \mathrm{~g}$ ), and $0.25 \mathrm{wt} \%$ SDS solution (12 g) was added to the first step swollen seed particles. The dosage of AgNPs varied from 0 to $35 \mathrm{wt} \%$ of St and DVB. The detailed formulas used to prepare the porous $\mathrm{P}(\mathrm{St}-\mathrm{DVB}) / \mathrm{AgNPs}$ composite microspheres were listed in Table 1. During the preparation of the separate emulsions, the AgNPs should be first dissolved in the mixture of styrene, DVB and porogen by using the ultrasonic disperse method. Toluene, $n$-hexadecane or $n$-heptane was used as porogen. The swollen seeds and monomer mixtures were stirred in the dark at room temperature for $12 \mathrm{~h}$. Then $2 \mathrm{~g}$ of $10 \mathrm{wt} \%$ polyvinylalcohol aqueous solution were added as a stabilizer and the solution was stirred for another hour. The mixture was purged with argon for $10 \mathrm{~min}$ and transferred into a water shaking bath, which was preheated to $70{ }^{\circ} \mathrm{C}$. The shaking rate was $150 \mathrm{rpm}$. The polymerization proceeded for $24 \mathrm{~h}$. Microspheres were isolated by centrifugation and by a Soxhlet extraction apparatus with water (24 h) and THF (24 h) in order to remove impurities. Finally, particles were dried in vacuum at $60{ }^{\circ} \mathrm{C}$ and a brownish powder was obtained with low density.

Table 1 Formulas of porous P(St-DVB)/AgNPs composite
microspheres

\begin{tabular}{llllll}
\hline & & St & DVB & Ag/(St + DVB $)$ & Ag/composite \\
& Porogen & feed $[\mathrm{g}]$ & $\begin{array}{l}\text { feed }[\mathrm{g}] \\
{[\mathrm{wt} \%]}\end{array}$ & \\
\hline S0 & Toluene & 0.60 & 0.60 & 0 & 0 \\
S1 & Toluene & 0.60 & 0.60 & 5 & 4.76 \\
S2 & Toluene & 0.60 & 0.60 & 15 & 13.0 \\
S3 & Toluene & 0 & 1.2 & 20 & 16.7 \\
S4 & Toluene & 0.20 & 1.0 & 20 & 16.7 \\
S5 & Toluene & 0.40 & 0.80 & 20 & 16.7 \\
S6 & Toluene & 0.60 & 0.60 & 20 & 16.7 \\
S7 & Toluene & 0.75 & 0.45 & 20 & 16.7 \\
S8 & Toluene & 0.86 & 0.34 & 20 & 16.7 \\
S9 & Toluene & 1.0 & 0.20 & 20 & 16.7 \\
S10 & Toluene & 1.2 & 0 & 20 & 16.7 \\
S11 & Toluene & 0.60 & 0.60 & 25 & 20.0 \\
S12 & Toluene & 0.60 & 0.60 & 35 & 25.9 \\
S13 & $n$-Hexadecane & 0.60 & 0.60 & 20 & 16.7 \\
S14 & $n$-Heptane & 0.60 & 0.60 & 20 & 16.7 \\
& & & & &
\end{tabular}

\subsection{Catalytic properties of porous $\mathrm{P}(\mathrm{St}-\mathrm{DVB}) / \mathrm{AgNPs}$ composite microspheres}

In a typical catalytic experiment, both aqueous solutions of the dye (MB, $\left.10 \mathrm{mg} \mathrm{L}^{-1}\right)$ and of $\mathrm{NaBH}_{4}\left(1 \mathrm{~mol} \mathrm{~L}^{-1}\right)$ were freshly prepared. Subsequently, a series of porous $\mathrm{P}(\mathrm{St}-\mathrm{DVB}) / \mathrm{AgNPs}$ composite microsphere samples $(10 \mathrm{mg})$ were mixed with $15 \mathrm{~mL}$ of $10 \mathrm{mg} \mathrm{L}^{-1} \mathrm{MB}$ aqueous solution, and $0.5 \mathrm{~mL}$ of $1 \mathrm{~mol} \mathrm{~L}^{-1} \mathrm{NaBH}_{4}$ solution was rapidly injected into this mixture while stirring. The color of the mixture vanished gradually, indicating the reduction of the dye. The concentration changes of $\mathrm{MB}$ were monitored with a UV-Vis spectrophotometer.

In order to investigate reusability of the $\mathrm{P}(\mathrm{St}-\mathrm{DVB}) / \mathrm{AgNPs}$ composite microsphere catalyst, the catalyst was separated from the reaction system by centrifugation (4000 rpm, $15 \mathrm{~min}$ )

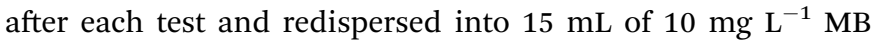
aqueous solution. The catalytic activity test was performed again under the same condition as described above. The reusability test was repeated for 10 times.

\subsection{Characterization}

2.6.1. Optical microscope (OM). The morphologies and microstructures of the polystyrene seeds in different swelling processes and of the prepared porous microspheres were directly observed using a Leica DMi8 A.

2.6.2. Scanning electron microscope (SEM). The morphologies of the polystyrene seeds and synthesized microspheres were characterized by an SEM (LEO1525). The dispersion of the AgNPs on the composite microspheres was detected by energydispersive X-ray spectroscopy (EDS), backscattering electro detector (BSED) and secondary electrons (SE).

2.6.3. Transmission electron microscope (TEM). The morphologies of the synthesized AgNPs and the AgNPs loaded on the composite spheres were characterized by a JEM-2100 (JEOL) TEM. The samples were dispersed in ethanol via ultrasonication at the concentration of $100 \mathrm{ppm}$ and dripped onto an ultrathin carbon $\mathrm{Cu}$ grid. The number average diameters $D_{\mathrm{n}}$ of all the particles were calculated by Image $\mathrm{J}$.

2.6.4. X-ray diffraction (XRD). The crystal structure of the synthesized AgNPs and the chemical composition of the composite microspheres were analyzed by an XRD (Rigaku D/ max-2550 PC X-ray diffractometer with $\mathrm{Cu}-\mathrm{K} \alpha$ radiation, wavelength $\lambda=1.5406 \AA$ ).

2.6.5. Thermogravimetric analysis (TGA). The thermostability of samples was evaluated in an atmosphere of air on a Mettler Toledo thermogravimetric analyzer. Each sample with a mass of $10 \mathrm{mg}$ was heated from 30 to $800{ }^{\circ} \mathrm{C}$ at a heating rate of $10{ }^{\circ} \mathrm{C} \mathrm{min}^{-1}$.

Specific surface areas and cumulative pore volumes were determined by measuring nitrogen adsorption on a porosity meter (Thermo Scientific, Surfer) and calculated using Brunauer-Emmett-Teller (BET) theory.

UV-Vis absorption spectra were used to characterize the concentration of $\mathrm{MB}$ during the photocatalysis degradation by UV-Vis spectrometer (Varian, Cary 50). 


\section{Results and discussion}

\subsection{Characterization of organic soluble silver nanoparticles}

The AgNPs used in this research work are organic-soluble AgNPs powders which were synthesized by the reduction of precursor $\mathrm{AgNO}_{3}$ with ascorbic acid in the presence of oleic acid and $n$-butylamine as surfactants. Fig. 1(a) shows the XRD pattern of the AgNPs powders. There are five diffraction peaks with $2 \theta$ values of about $38.1^{\circ}, 44.3^{\circ}, 64.4^{\circ}, 77.5^{\circ}$ and $81.6^{\circ}$ in agreement with the silver (JCPDS no. 04-0783) ${ }^{36}$ values for a facecentered cubic (FCC) crystal structure corresponding to (111), (200), (220), (311) and (222) lattice planes, respectively. Fig. 1(b) shows a TEM image of the uniform and small nanoparticles of the AgNPs. The size distribution of synthesized AgNPs in the right bottom corner illustrated an average size of $5.61 \pm 0.55 \mathrm{~nm}$ in diameter.

The synthesized AgNPs can be well dispersed in organic solvents to be transparent solutions in low concentration. In Fig. 2(a-e) $0.1 \mathrm{mg}$ AgNPs powders are dissolved in $5 \mathrm{~mL}$ different organic solvents which were used as monomer, crosslinker or porogen in the synthesis of $\mathrm{P}(\mathrm{St}-\mathrm{DVB}) / \mathrm{AgNPs}$ composite microspheres. In the mixture solution of toluene and water, the AgNPs are well dissolved in toluene and two layers were formed, where

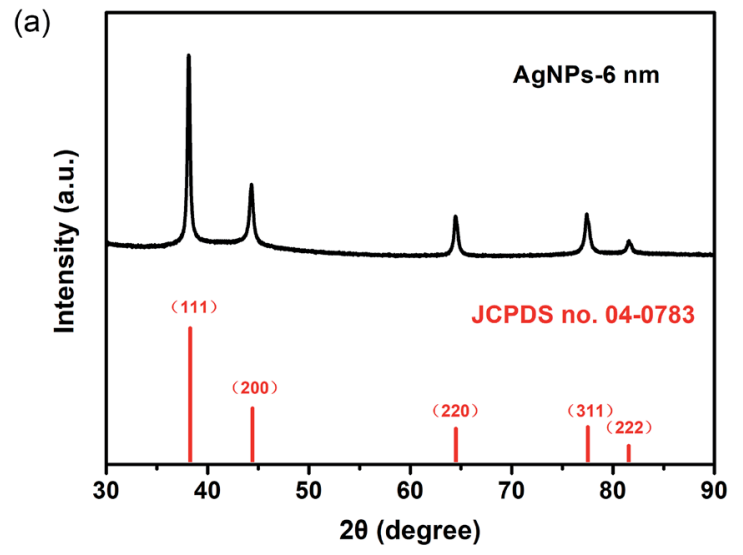

(b)

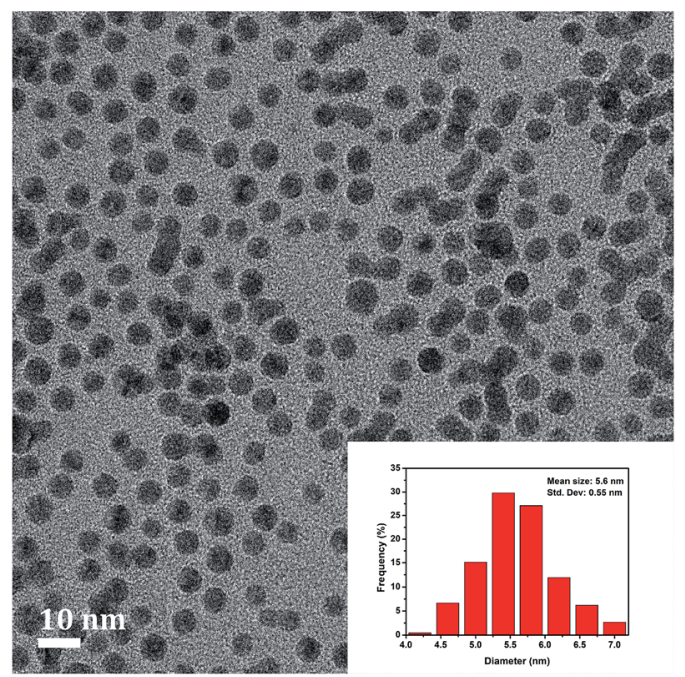

Fig. 1 (a) XRD pattern of the synthesized AgNPs. (b) TEM image of the AgNPs. Inset shows the size distribution of the AgNPs. toluene is in the upper layer (Fig. 2(f)). So the AgNPs have good solubility in the organic phase during the polymerization.

\subsection{The formation of the porous $\mathrm{P}(\mathrm{St}-\mathrm{DVB}) / \mathrm{AgNPS}$ composite microspheres}

As illustrated in Fig. 3, seed swelling polymerization method was used to prepare the porous $\mathrm{P}(\mathrm{St}-\mathrm{DVB}) / \mathrm{AgNPs}$ composite microspheres. During the polymerization, linear polystyrene particles in Fig. 3(a) are worked as templates. The formation process of composite microspheres should first undergo the swelling of seed particles with activator in Fig. 3(b); then the second step swelling to absorb monomer, crosslinker, initiator, porogen and AgNPs into the polystyrene seeds. At last, the polymerization reacts inside the seeds to get the porous composite microspheres in Fig. 3(c). In the previous reports, the size and size distribution of the final composite microspheres were completely determined by the initial polystyrene seed particles, ${ }^{37,38}$ so the seeds need not only to be monodisperse, but also to be non-crosslinked to allow reactants going into the

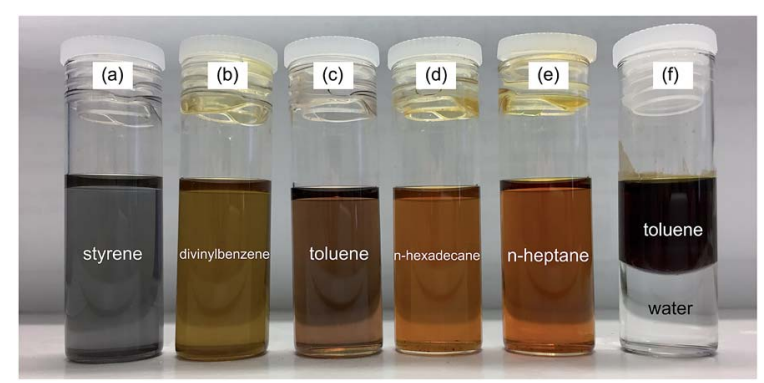

Fig. 2 Photographs of synthesized AgNPs redispersed in (a) styrene, (b) divinylbenzene, (c) toluene, (d) $n$-hexadecane, (e) $n$-heptane and mixture ( $f$ ) of toluene (upper) and water (bottom) (1: 1 by volume). The concentration of AgNPs is $20 \mathrm{mg} \mathrm{L}^{-1}$ in (a-e) and the concentration of AgNPs is $100 \mathrm{mg} \mathrm{L}^{-1}$ in (f).

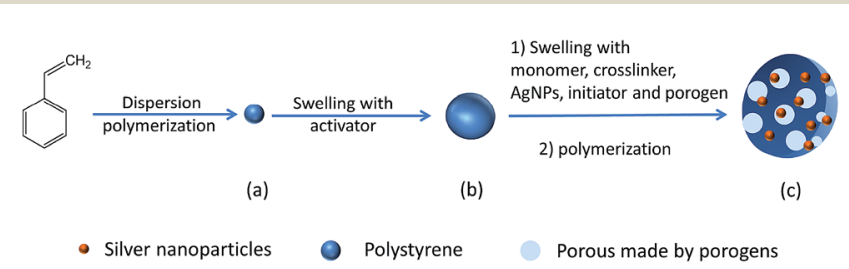

Fig. 3 The scheme of the formation process of porous P(St-DVB)/ AgNPs composite microspheres.
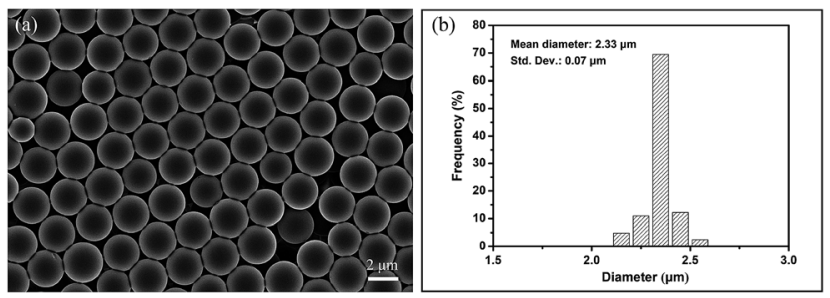

Fig. 4 (a) SEM image and (b) size distribution of polystyrene seeds. 

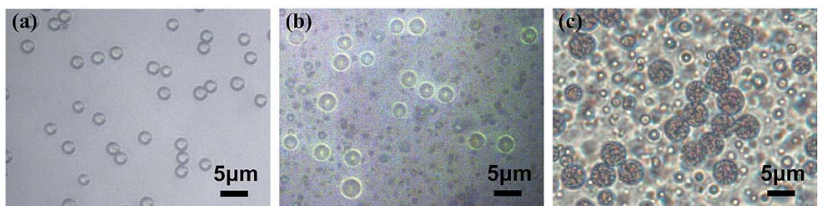

Fig. 5 Optical microscope images for the preparation of the porous $\mathrm{P}(\mathrm{St}-\mathrm{DVB}) /$ AgNPs composite microspheres (S12 in Table 1, St feed $0.60 \mathrm{~g}$, DVB feed $0.60 \mathrm{~g}$, toluene, $35 \mathrm{wt} \% \mathrm{Ag} /(\mathrm{St}+\mathrm{DVB}))$. (a) Seed particles swelled with an activator in the first step swelling for $5 \mathrm{~h}$. (b) Seed particles swelled with St, DVB, AgNPs, toluene and DBPO in a second step swelling for $12 \mathrm{~h}$. (c) Synthesized porous P(St-DVB)/ AgNPs composite microspheres were obtained.

particles during the swelling stage. Here, precipitation polymerization of styrene seeds was performed in ethanol with AIBN as initiator, polyvinylpyrrolidone (PVP-K30) and Aliquat 336 as stabilizers. Following polymerization overnight and purification by intense washing, the resulting seed particles were characterized by SEM (Fig. 4(a)). The SEM picture shows the smooth spherical monodisperse particles with an average diameter of $2.33 \pm 0.07 \mu \mathrm{m}$ in Fig. 4(b). The size and size distribution proved to be highly reproducible throughout several runs.

During the formation processes of porous $\mathrm{P}(\mathrm{St}-\mathrm{DVB}) / \mathrm{AgNPS}$ composite microspheres, microscope images are used to record the microstructure of the particles in different reaction steps. In Fig. 5(a) the swollen seed particles with an activator are shown after 5 hours' swelling. As illustrated in Fig. 3(b), the particles are sphere and monodisperse in size. The average size of the seed particles is $2.34 \mu \mathrm{m}$. In the following swelling process, the swollen seed particles were growing. Fig. 5(b) is a microscope picture of polystyrene seeds swelled with monomer, crosslinker, AgNPs, porogen and initiator in the second swelling step. The average particle size in the second swelling step grows up to $3.38 \mu \mathrm{m}$, which means the monomer, AgNPs, porogen and initiator went into the seed particles by permeation force. ${ }^{39}$ By increasing the temperature of the water shaking bath to the reaction temperature, the crosslinking reaction took place inside the swollen polystyrene seeds. In Fig. 5(c), the morphology of the final P(St-DVB)/AgNPs composite microspheres after $24 \mathrm{~h}$ crosslinking reaction is shown. Compared with the polystyrene seed particles and the particles after the second step swelling in Fig. 3(a and b), the porous structures can be clearly observed in the final microspheres (Fig. 3(c)). The average size of the obtained porous $\mathrm{P}(\mathrm{St}-$ DVB)/AgNPs composite is $4.5 \mu \mathrm{m}$.

There are several methods for depositing AgNPs onto the surface of polystyrene matrix, such as in situ metals reduction, ${ }^{\mathbf{4 0}}$ electrostatic deposition ${ }^{41}$ and layer-by-layer assembly. ${ }^{42}$ Usually, by the methods mentioned above to prepare the porous composite microspheres, the polymer matrix should be first synthesized and then the silver nanoparticles attached onto the surfaces. The processes are complicated and time-consuming.

Here, organic soluble AgNPs were directly mixed with monomer during the polymerization to load onto porous polymer matrix. The morphology and the distribution of AgNPs on porous $\mathrm{P}(\mathrm{St}-\mathrm{DVB})$ were characterized by TEM, EDS and XRD. S12 in Table 1 (St feed $0.60 \mathrm{~g}$, DVB feed $0.60 \mathrm{~g}$, toluene, $35 \mathrm{wt} \% \mathrm{Ag} /$ (St + DVB)) was chosen as a standard sample. TEM picture, Fig. 6(a) shows a whole microsphere. Referring to the thin part of the microsphere in Fig. 6(b) and the cross section in Fig. 6(c), the deposited AgNPs are nearly spherical and well separated from each other. The average diameter of AgNPs in Fig. 6(d) is $20.97 \pm 4.31 \mathrm{~nm}$ which is calculated based on statistical diameter of more than 100 nanoparticles measured from Fig. 6(c). In order to prove the AgNPs were loaded inside the microspheres, energy-dispersive X-ray spectroscopy (EDS) was carried out on the cross section of the composite microspheres.
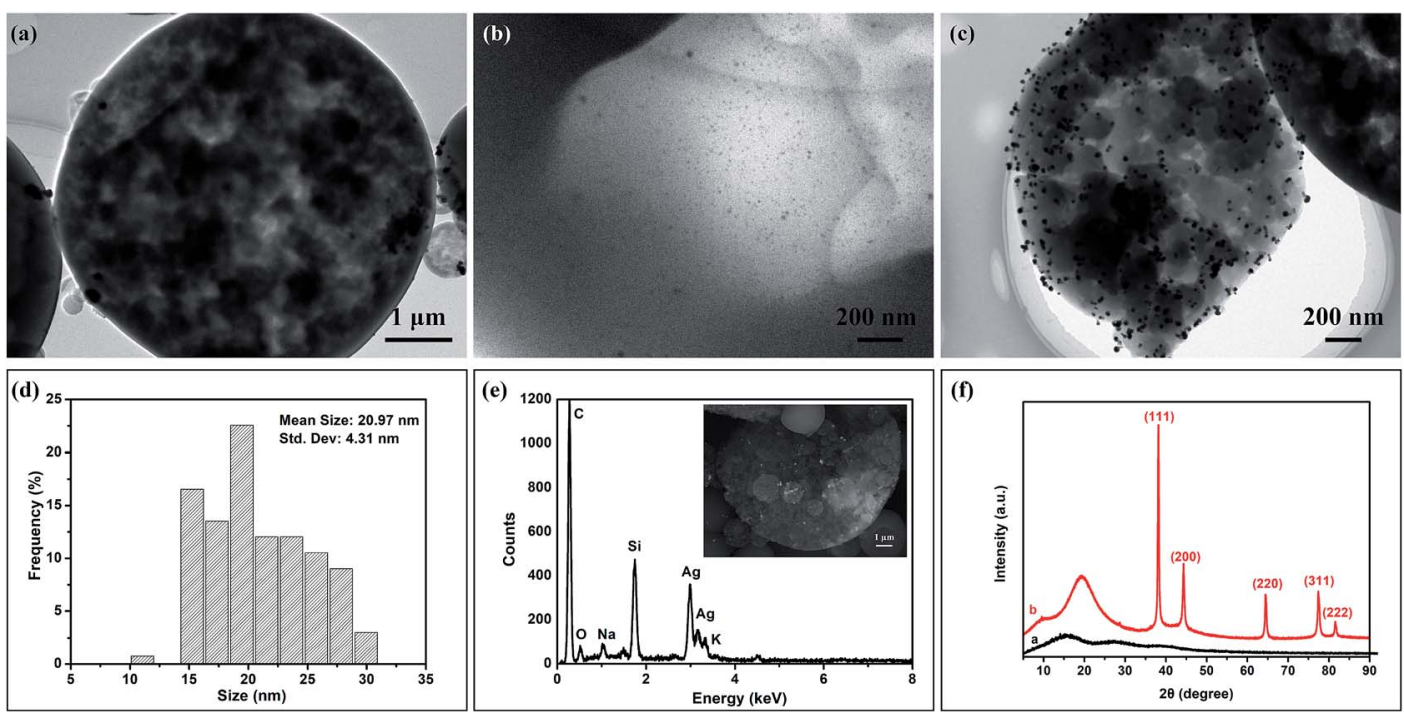

Fig. 6 TEM images of porous P(St-DVB)/AgNPs composite microspheres (S12 in Table 1, St feed $0.60 \mathrm{~g}$, DVB feed $0.60 \mathrm{~g}$, toluene, $35 \mathrm{wt} \%$ Ag/(St + DVB)): (a) the whole microsphere, (b) the thin part and (c) the cross-section of the composite microsphere, (d) the particle size distribution of AgNPs calculated from (c), (e) EDS image corresponding to the inserted SE (second electron) image on the cross section of the microsphere, (f) XRD patterns of P(St-DVB) microsphere without AgNPs (SO, curve a) and porous P(St-DVB)/AgNPs composite microspheres (S12, curve b). 

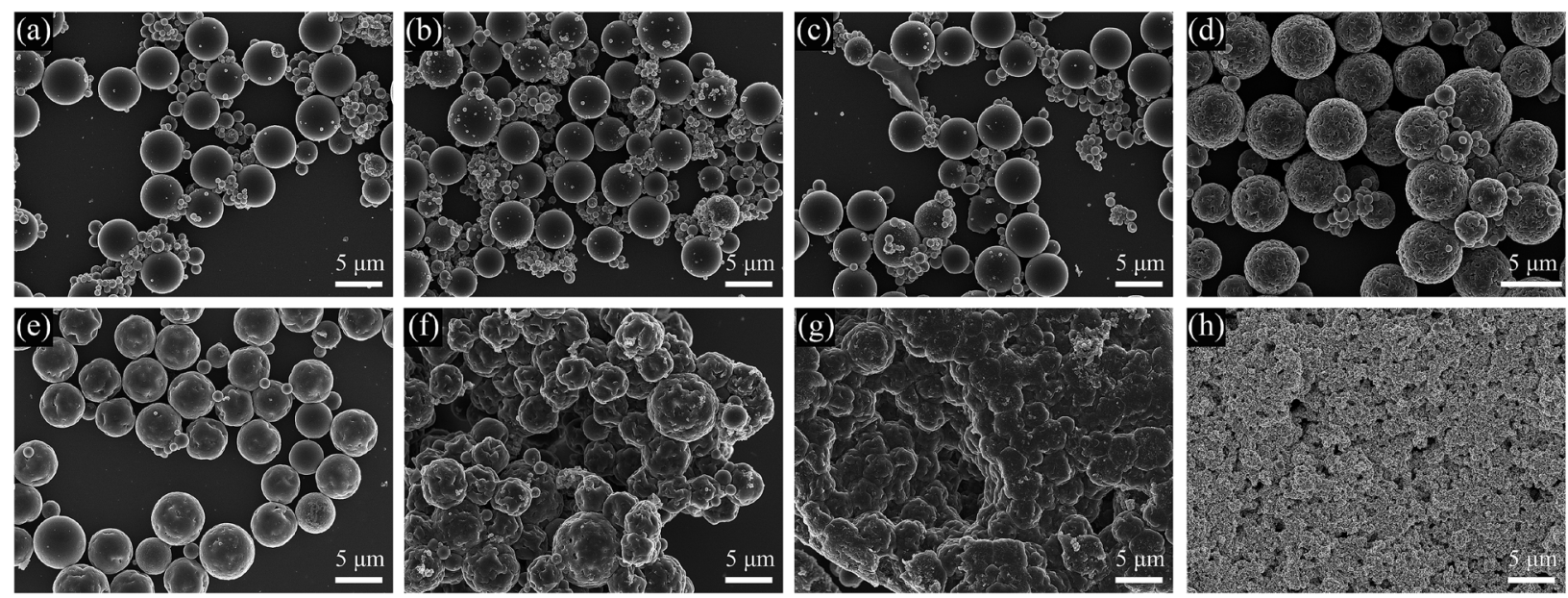

Fig. 7 SEM pictures of porous P(St-DVB)/AgNPs composite microspheres with different St and DVB ratio. (a) is corresponding to sample 3 (St 0 , DVB $1.2 \mathrm{~g}$ ), (b) is corresponding to sample 4 (St $0.2 \mathrm{~g}$, DVB $1.2 \mathrm{~g}$ ), (c) is corresponding to sample 5 (St $0.4 \mathrm{~g}$, DVB $0.8 \mathrm{~g}$ ), (d) is corresponding to sample 6 (St $0.6 \mathrm{~g}$, DVB $0.6 \mathrm{~g}$ ), (e) is corresponding to sample 7 (St $0.75 \mathrm{~g}, \mathrm{DVB} 0.45 \mathrm{~g}$ ), (f) is corresponding to sample 8 (St $0.86 \mathrm{~g}, \mathrm{DVB} 0.34 \mathrm{~g}$ ), (g) is corresponding to sample 9 (St $1.0 \mathrm{~g}$, DVB $0.2 \mathrm{~g}$ ), and (h) is corresponding to sample 10 (St $1.2 \mathrm{~g}$, DVB 0).

Thus Fig. 6(e) apparently illustrated $\mathrm{Ag}$ atomic peaks. Compared with blank sample S0, XRD pattern of S12 in Fig. 6(f) shows clear $2 \theta$ values of $38.1^{\circ}, 42.1^{\circ}, 64.5^{\circ}, 77.5^{\circ}$ and $81.6^{\circ}$ which corresponding to the synthesized AgNPs powders in Fig. 1(a) and the peak at approximately $20^{\circ}$ comes from the amorphous $\mathrm{P}(\mathrm{St}-\mathrm{DVB})$. These results confirm that synthesized AgNPs had been successfully mono dispersed both inside and on the surface of the composite microspheres.

3.2.1. Effect of St/DVB on morphology of P(St-DVB)/AgNPs composite microspheres. The porous $\mathrm{P}(\mathrm{St}-\mathrm{DVB}) / \mathrm{AgNPS}$ composite microspheres with different monomer (St) and crosslinker (DVB) ratios were synthesized while the amounts of AgNPs were kept constant and toluene was used as the porogen. The different ratios of St and DVB are shown in Table 1 from S3 to S10 in detail. Analysis by SEM indicated different morphologies for S3-S10, see Fig. 7, and suggested that the amount of DVB in the monomer and crosslinker feed did not only influence the size of microsphere but also the morphology. With high DVB feed, S3 (Fig. 7(a), St 0 g, DVB 1.2 g), S4 (Fig. 7(b), St $0.2 \mathrm{~g}$, DVB $1.0 \mathrm{~g}$ ) and S5 (Fig. 7(c), St $0.4 \mathrm{~g}$, DVB $0.8 \mathrm{~g}$ ) had almost smooth surfaces with similar average diameters of $4.5 \pm 0.7 \mu \mathrm{m}$, $4.4 \pm 0.6 \mu \mathrm{m}$ and $4.5 \pm 0.7 \mu \mathrm{m}$, respectively. When the St and DVB ratio was $1: 1$, S6 (Fig. 7(d), St $0.6 \mathrm{~g}$, DVB $0.6 \mathrm{~g}$ ), the diameter of the microspheres was still $4.5 \pm 0.7 \mu \mathrm{m}$, but the porous structures could be clearly seen on the surface of the microspheres. By decreasing the DVB amount further, as in S7 (Fig. 7(e), St 0.75 g, DVB 0.45 g), S8 (Fig. 7(f), St 0.86 g, DVB
$0.34 \mathrm{~g}$ ) and sample 9 (Fig. 7(g), St $1.0 \mathrm{~g}$, DVB $0.2 \mathrm{~g}$ ), the particles were not spheres anymore and started to aggregate and collapse. In S10 (Fig. 7(h), St $1.2 \mathrm{~g}$, DVB $0 \mathrm{~g}$ ), when the reaction system operated without any crosslinker, there were not any microspheres existing in the final product. The average diameters and specific surface areas of S3-S10 are listed in Table 2, the result indicates a well reproducible size range independent of monomer feed ratio. In contrast to diameters, the porosities of the composite microspheres were varied significantly with the DVB feed content. Specific surface areas of microspheres determined by nitrogen adsorption had impressive downtrend from $130.6 \mathrm{~m}^{2} \mathrm{~g}^{-1}$ to $38.8 \mathrm{~m}^{2} \mathrm{~g}^{-1}$ with decreasing DVB amount as shown in Table 2.

In summary, the composite microspheres with different morphologies range from smooth surface, porous, collapse to totally covered by AgNPs can be obtained by decreasing the amounts of DVB in the mixture of St and DVB. This is because a higher content of divinyl crosslinker would get more highly cross-linked polymers in the early stages of the polymerization process. ${ }^{43}$ The early and highly crosslinked nuclei had a limited adsorption of monomers and porogens. So smaller voids and relatively smooth surfaces appear, which corresponds to higher specific surface areas. ${ }^{44}$ Without sufficient DVB as crosslinker, the construction of a network of the microspheres failed and the spheres easily collapsed as in Fig. 7(f-h). In further, without crosslinker, no spheres existed anymore and the AgNPs were aggregated.

Table 2 The diameter and specific surface area of the synthesized porous composite microspheres with different monomer (St) and crosslinker (DVB) feed ratio

\begin{tabular}{|c|c|c|c|c|c|c|c|c|}
\hline & S3 & S4 & S5 & S6 & S7 & S8 & S9 & S10 \\
\hline$D_{\mathrm{n}}{ }^{a}(\mu \mathrm{m})$ & $4.5 \pm 0.7$ & $4.4 \pm 0.6$ & $4.5 \pm 0.7$ & $4.5 \pm 0.7$ & $4.7 \pm 1.0$ & $3.8 \pm 1.0$ & Aggl. & None \\
\hline$S^{b}\left(\mathrm{~m}^{2} \mathrm{~g}^{-1}\right)$ & 130.6 & 98.2 & 70.8 & 55.2 & 38.8 & - & - & - \\
\hline
\end{tabular}

${ }^{a}$ Number-average particle diameter calculated from the SEM pictures by Image-J software; standard deviation from measuring at least 100 particles.

${ }^{b}$ Specific surface area from BET nitrogen adsorption. 

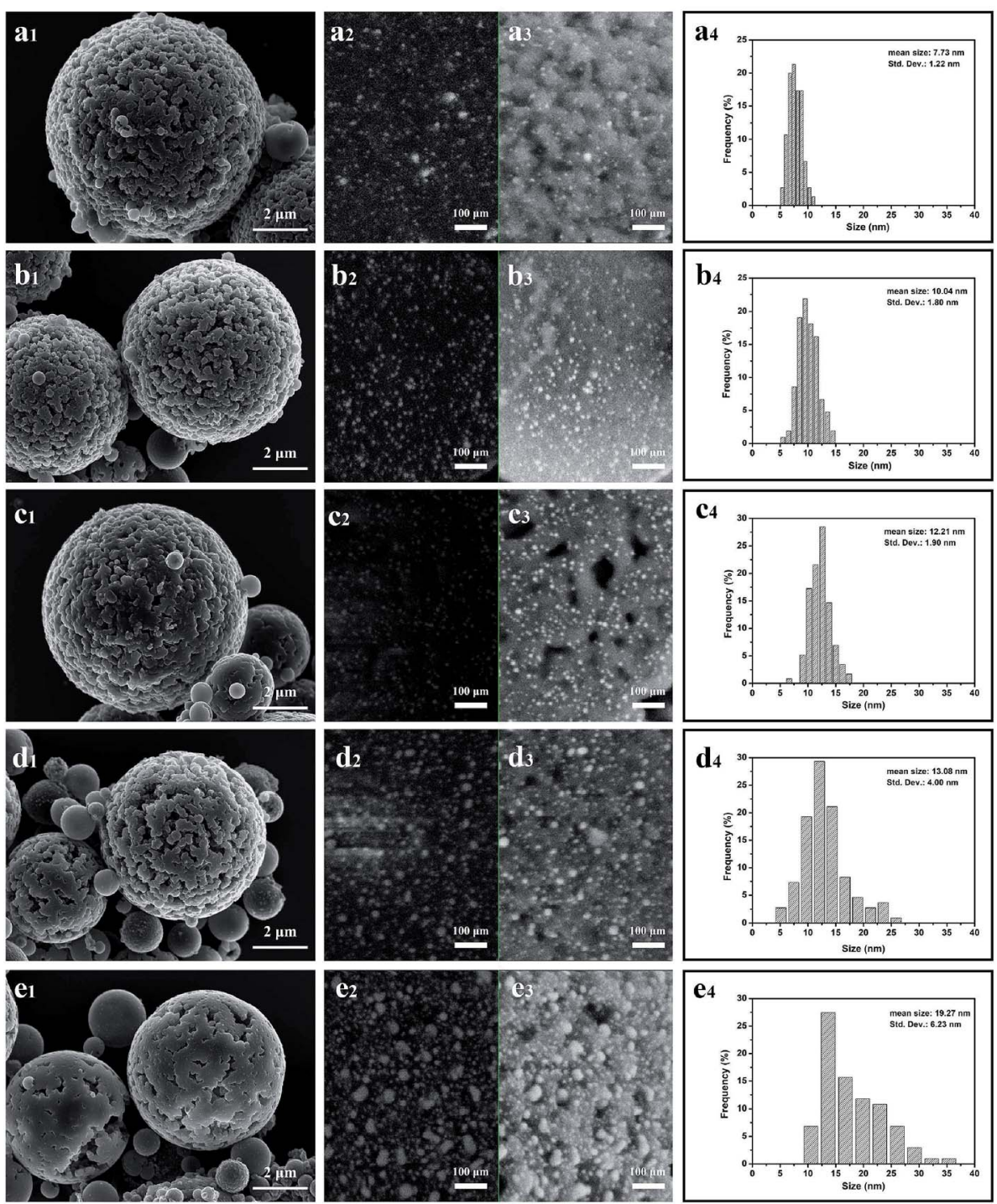

Fig. 8 The SEM images (series 1), BSED images (series 2), SE images (series 3) and size distribution of AgNPs (series 4) loaded on the porous P(StDVB)/AgNPs composite microspheres calculated from SE images with different AgNPs contents. (a1-a4) 4.76\% Ag/polymer matrix (sample S1), (b1-b4) 13.0\% Ag/polymer matrix (sample S2), (c1-c4) 16.7\% Ag/polymer matrix (sample S6), (d1-d4) 20\% Ag/polymer matrix (sample S11), (e1e4) $25.9 \%$ Ag/polymer matrix (sample S12).

3.2.2. Effect of concentration of AgNPs added in the polymerization on the morphology and AgNPs loading of composite microspheres. The concentration of silver precursor is crucial to the deposition of AgNPs on substrates by wet impregnation method to synthesize composite microspheres. ${ }^{45}$ The effect of concentration of organic-soluble AgNPs powders added in the swelling and polymerization process on the deposition of AgNPs on P(St-DVB) substrates were also investigated in this work. As the addictive ratio of St and DVB of $1: 1$ had the best porous structure, the addictive ratio of St and DVB of $1: 1$ and toluene as porogen were chosen to investigate the AgNPs loading states by adjusting the dosage of AgNPs in the polymerization. The detailed formulas are listed in Table 1 (S1, S2, S6, S11 and S12). In Fig. 8, SEM images were collected to illustrate the morphologies of the composite microspheres and BSED and SE images were taken to indicate the spread of
AgNPs on the surface of $\mathrm{P}(\mathrm{St}-\mathrm{DVB})$ substrates with different AgNPs additions in the polymerization. In Fig. 8(a2-e2 and a3-e3), the white and bright particles are silver nanoparticles loaded on the composite microspheres. The size distributions and average sizes of the loaded AgNPs are demonstrated in Fig. 8(a4-e4), calculated by Image J from Fig. 8(a3-e3). The SE and BSED images show that the AgNPs were well dispersed on the surfaces of the composite microspheres. However, the average sizes of the AgNPs in Fig. 8(a4-e4) increased from $7.73 \pm 1.22 \mathrm{~nm}$ to $19.27 \pm 6.23 \mathrm{~nm}$ with increasing the additive amount of AgNPs, i.e. the added AgNPs tended to aggregate during the polymerization compared with the original single AgNPs in Fig. 1. Moreover, with increasing AgNPs addition, the size distributions of AgNPs loaded on the composite microspheres went broader due to the various aggregation rate caused by different areas. 
Table 3 The specific surface area, pore volume and pore size characteristics of the porous $\mathrm{P}(\mathrm{St}-\mathrm{DVB}) / \mathrm{AgNPs}$ composite microspheres with different AgNPs contents

\begin{tabular}{llll}
\hline Samples & $S_{\mathrm{BET}}{ }^{a}\left[\mathrm{~m}^{2} \mathrm{~g}^{-1}\right]$ & $V_{\text {total }}^{b}\left[\mathrm{~cm}^{3} \mathrm{~g}^{-1}\right]$ & Pore size $^{c}[\mathrm{~nm}]$ \\
\hline Pure PS (S0) & 34.47 & 0.1683 & 86.49 \\
$4.76 \% \mathrm{Ag}$ (S1) & 41.96 & 0.2155 & 69.99 \\
$13.0 \% \mathrm{Ag}$ (S2) & 53.99 & 0.1713 & 44.87 \\
$16.7 \% \mathrm{Ag}$ (S6) & 55.16 & 0.1940 & 24.85 \\
$20.0 \% \mathrm{Ag}$ (S11) & 37.84 & 0.1570 & 51.86 \\
$25.9 \% \mathrm{Ag}$ (S12) & 31.41 & 0.1224 & 55.27
\end{tabular}

${ }^{a}$ Specific surface area from BET nitrogen adsorption. ${ }^{b}$ Cumulative pore volume from BET nitrogen adsorption. ${ }^{c} \mathrm{BJH}$ desorption average pore diameter.

In order to get further insight into the effects of AgNPs addictive amounts on the morphologies of the porous $\mathrm{P}(\mathrm{St}$ DVB)/AgNPs composite microspheres, the specific surface areas were calculated from nitrogen adsorption/desorption isotherms by utilizing the BET method. The total volume and the corresponding pore size were determined by the BarrettJoyner-Halenda (BJH) from inverting the desorption branch of porous $\mathrm{P}(\mathrm{St}-\mathrm{DVB}) / \mathrm{AgNPs}$ composite microspheres with different AgNPs contents, see Table 3 . The pore sizes of the porous P(StDVB)/AgNPs composite microspheres first decreased then increased with increasing the additional concentration of AgNPs, while the specific surface areas and pore volumes had the inverse trend of the pore size. These could be the reason that the first adding of AgNPs occupied some of the pores and then the aggregated bigger AgNPs occupied in the pores causing the pore to be expanded.

TGA of the pristine porous $\mathrm{P}(\mathrm{St}-\mathrm{DVB})$ microspheres and porous $\mathrm{P}(\mathrm{St}-\mathrm{DVB}) / \mathrm{AgNPs}$ composite microspheres were performed in an air atmosphere to analyze the effect of AgNPs concentration on thermal degradation and to calculate the

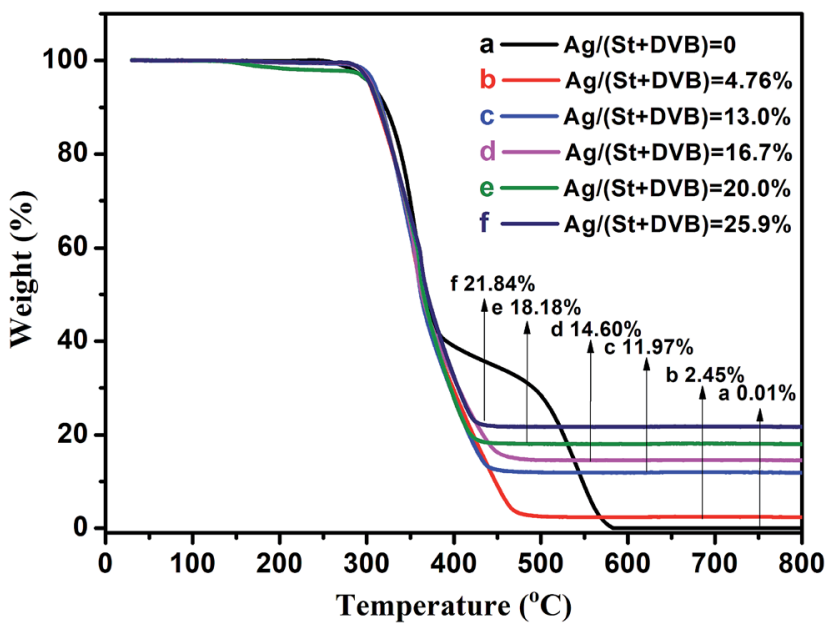

Fig. 9 TGA curves of porous P(St-DVB)/AgNPs composite microspheres with different AgNPs contents (a) pure PS microspheres, (b) 4.76\% AgNPs, (c) $13.0 \%$ AgNPs, (d) $16.7 \%$ AgNPs, (e) $20.0 \%$ AgNPs, (f) $25.9 \%$ AgNPs. amount of AgNPs loaded on the porous P(St-DVB)/AgNPs composite microspheres. Residual weight of pristine porous $\mathrm{P}(\mathrm{St}-\mathrm{DVB})$ microspheres at $800{ }^{\circ} \mathrm{C}$ is $0.01 \%$, while residual weight of the $\mathrm{P}(\mathrm{St}-\mathrm{DVB}) / \mathrm{AgNPs}$ composite microspheres varies with the theoretical AgNPs addictive amount of $4.76 \%, 13.0 \%$, $16.7 \%, 20.0 \%$ and $25.9 \%$ are $2.45 \%, 11.97 \%, 14.60 \%, 18.18 \%$ and $21.84 \%$. The tested residual weights were less than the real added weights of AgNPs, suggesting that not all the AgNPs added during the polymerization can be loaded on the composite microspheres. There were still some AgNPs left in the solution and washed away by THF (Fig. 9).

3.2.3. Effect of porogens on the morphology and AgNPs loading of $\mathbf{P}(\mathrm{St}-\mathrm{DVB}) / \mathbf{A g N P s}$ composite microspheres. The morphology of the composite microspheres was not only influenced by the St/DVB ratio and the dosage of AgNPs in the polymerization, but also affected by the porogens used during the synthesis. ${ }^{25,46}$ Here, three different porogens - toluene, $n$ hexadecane and $n$-heptane were chosen to investigate the influence of variety of porogens on porous structures of the composite microspheres.

Regarding the SEM images in Fig. 10 (series 1) and size distribution in Fig. 10 (series 2) of the $\mathrm{P}(\mathrm{St}-\mathrm{DVB}) / \mathrm{AgNPS}$ composite microspheres, the composite microspheres have more porosities both inside and on the surface when toluene (Fig. 10(a)) and $n$-hexadecane (Fig. 10(b)) were used as porogens. While the interior of the composite microspheres is porous and surface is smooth with $n$-heptane used as porogen (Fig. 10(c)). The differences of porosities could be attributed to the formation mechanism of the pores by different porogens. When toluene was used as the porogen during the polymerization, the discrete phase consisted of styrene, DVB, DBPO, AgNPs and toluene, whereas the continuous phase was an aqueous poly(vinylalcohol) solution, because toluene is a thermodynamically good solvent for the final crosslinked microspheres, which can easily be swollen in it. Inside every discrete polystyrene seed template, a continuous network grew by adding monomers. After a certain time, the network became incapable of absorbing more toluene due to an increasing amount of crosslinking. A precipitation occurred at this moment, which was after the gelation point of the network. It is this phase separation that yields the porosity of the microspheres. ${ }^{25,47}$ Usually in such a condition, micropores and mesopores are predominant, resulting in particles with high surface area values but low pore volumes. ${ }^{48}$ On the other hand, if a nonsolvent for the $\mathrm{P}(\mathrm{St}-\mathrm{DVB})$ was used as a porogen, such as $n$ hexadecane and $n$-heptane instead of toluene as in the previous case, pore formation occurred in a different way. In this case, phase separation occurred before the gelation point, since hexadecane and heptane cannot swell the growing polymer chains. At the start of the initiation, separated smaller particles of polymer nuclei grew in a discontinuous phase inside every discrete monomer phase droplet. These nuclei agglomerate was formed via inter-nuclei crosslinking and the final porous particle was produced. ${ }^{49}$ In contrast to using toluene as porogens, macropores are predominant, resulting in particles with a significantly lower surface area but larger pore volumes. However, since $n$-heptane is still a non-solvent for $\mathrm{P}(\mathrm{St}-\mathrm{DVB})$ polymer, it has not any pores on the surface. However, pores existed inside the microspheres. 

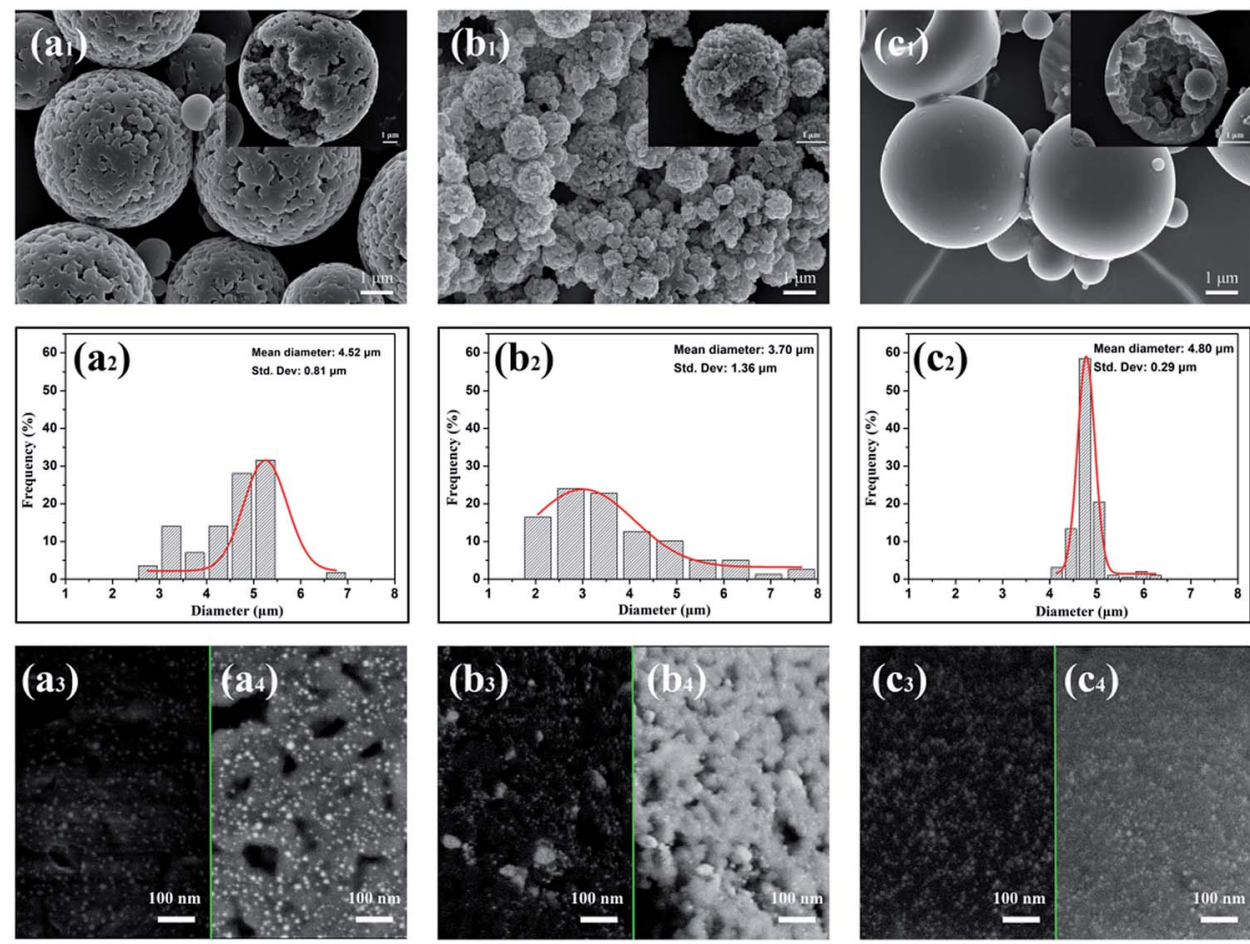

Fig. 10 SEM images (series 1), size distribution (series 2) and BSED and SE images of AgNPs loading (series 3 and 4 ) of the porous P(St-DVB)/ AgNPs composite microspheres using different porogens toluene (series a), $n$-hexadecane (series b) and $n$-heptane (series $c$ ). The insert images of SEM images in series 1 are the corresponding cross-sections of the composite microspheres.

Referring to the previous literature, ${ }^{50}$ this is due to the differences in solubility parameters between nonsolvent and polymer. When the difference in the solubility parameter is too large, a "skin" formation is promoted. The difference in the solubility parameter was increasing by a decreasing polarity of porogen. Since the continuous phase was water in our polymerization system, highly nonpolar porogen such as $n$-heptane opposed to be present in the water/oil interface due to the high interfacial tension. The interface became rich in St monomer and $\mathrm{P}(\mathrm{St}-\mathrm{DVB})$ polymer, resulting in a "skin" layer, while the interior was porous. Regarding the size distribution of microspheres in Fig. 10 (series 2), toluene and $n$-heptane used as porogens tend to obtain monodisperse composite microspheres, while the size distribution of the microspheres is wider using $n$-hexadecane.
Furthermore, the porosity parameters of $\mathrm{P}(\mathrm{St}-\mathrm{DVB}) / \mathrm{AgNPs}$ composite microspheres synthesized using different porogens were studied by sorption analysis using nitrogen as the sorbate molecule. Nitrogen adsorption-desorption isotherms and corresponding pore size distribution of $\mathrm{P}(\mathrm{St}-\mathrm{DVB}) / \mathrm{AgNPs}$ composite microspheres are shown in Fig. 11. The microspheres, which were obtained using toluene as the porogen exhibited a combination of type III and type IV nitrogen sorption isotherms and the $\mathrm{H} 3$ type of hysteresis loop seen from the IUPAC Technical Report, suggesting the presence of mesopores and a small number of macropores. ${ }^{51}$ The microspheres, which were obtained using $n$-hexadecane as the porogen, exhibited type III nitrogen sorption isotherms and $\mathrm{H} 3$ type of hysteresis loop, suggested the presence of micropores and macropores.
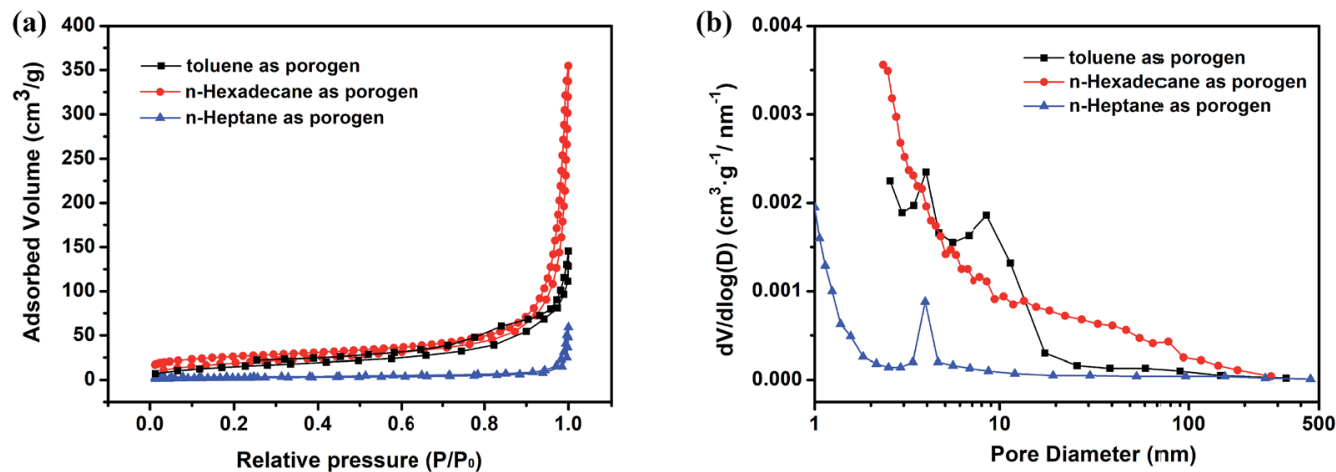

Fig. 11 (a) Nitrogen adsorption-desorption isotherms and (b) the corresponding pore size distribution for P(St-DVB)/AgNPs composite microspheres with different porogens. 
Table 4 The specific surface area and pore size characteristics of the porous $\mathrm{P}(\mathrm{St}-\mathrm{DVB}) / \mathrm{AgNPs}$ composite microspheres synthesized by different porogens

\begin{tabular}{lccc}
\hline Samples & $S_{\text {BET }}{ }^{a}\left[\mathrm{~m}^{2} \mathrm{~g}^{-1}\right]$ & $V_{\text {total }}^{b}\left[\mathrm{~cm}^{3} \mathrm{~g}^{-1}\right]$ & Pore size $^{c}[\mathrm{~nm}]$ \\
\hline Toluene (S6) & 55.16 & 0.1940 & 24.85 \\
$n$-Hexadecane (S13) & 70.80 & 0.5416 & 75.46 \\
$n$-Heptane (S14) & 8.65 & 0.0746 & 3.91
\end{tabular}

${ }^{a}$ Specific surface area from BET nitrogen adsorption. ${ }^{b}$ Cumulative pore volume from BET nitrogen adsorption. ${ }^{c} \mathrm{BJH}$ desorption average pore diameter.

For the microspheres prepared with $n$-heptane, the results from the curves showed that they only had small amount of micropores or mesopores on the surface of the microspheres. The detailed surface area and pore size parameters listed in Table 4 indicated that $n$-hexadecane as a porogen made macropores and $n$-heptane nearly had not any contribution to the pore surface, which were correlated with SEM images in Fig. 10.

In order to analyze the influence of the porogens used on the loading of AgNPs, TGA curves of different microspheres obtained by the different porogens were performed in air atmosphere. The obtained TGA curves showed that the microspheres using toluene as a porogen contained the most amount of AgNPs, while the microspheres using $n$-heptane as a porogen contained the least amount of AgNPs. This could be related to the morphologies of the obtained microspheres. The distribution of AgNPs on the surfaces of the microspheres was also investigated. The SE and BSED images in Fig. 10 (series 3) showed silver nanoparticles, which were deposited uniformly along the outer surfaces of the microspheres. The sizes of the silver nanoparticles followed the order: $\mathrm{S} 13$ ( $n$-hexadecane as porogen) $>\mathrm{S} 6$ (toluene as porogen) > S14 (n-heptane as porogen).

\subsection{Catalytic activity of porous $\mathbf{P}(\mathrm{St}-\mathrm{DVB}) / \mathrm{AgNPs}$ composite microspheres}

Metallic silver nanoparticles have been extensively investigated for their catalytic activities for degradation of nitrophenols,

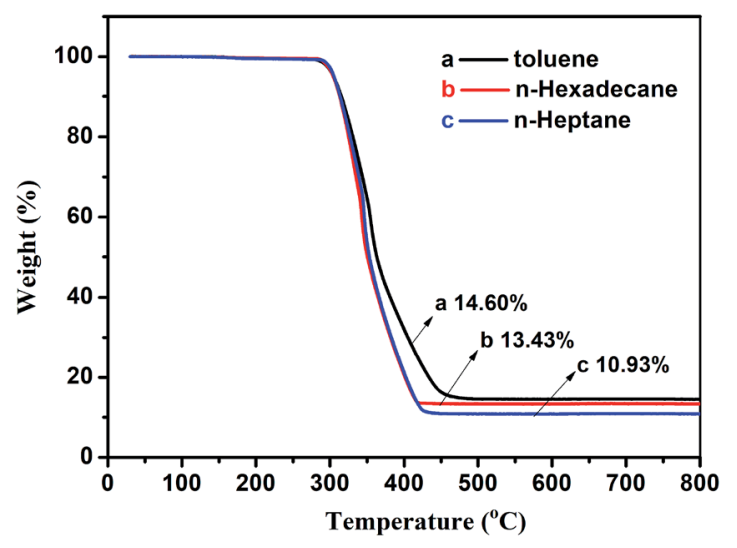

Fig. 12 TGA curves of porous P(St-DVB)/AgNPs composite microspheres synthesized by different porogens (a) toluene, (b) $n$-hexadecane and (c) $n$-heptane.
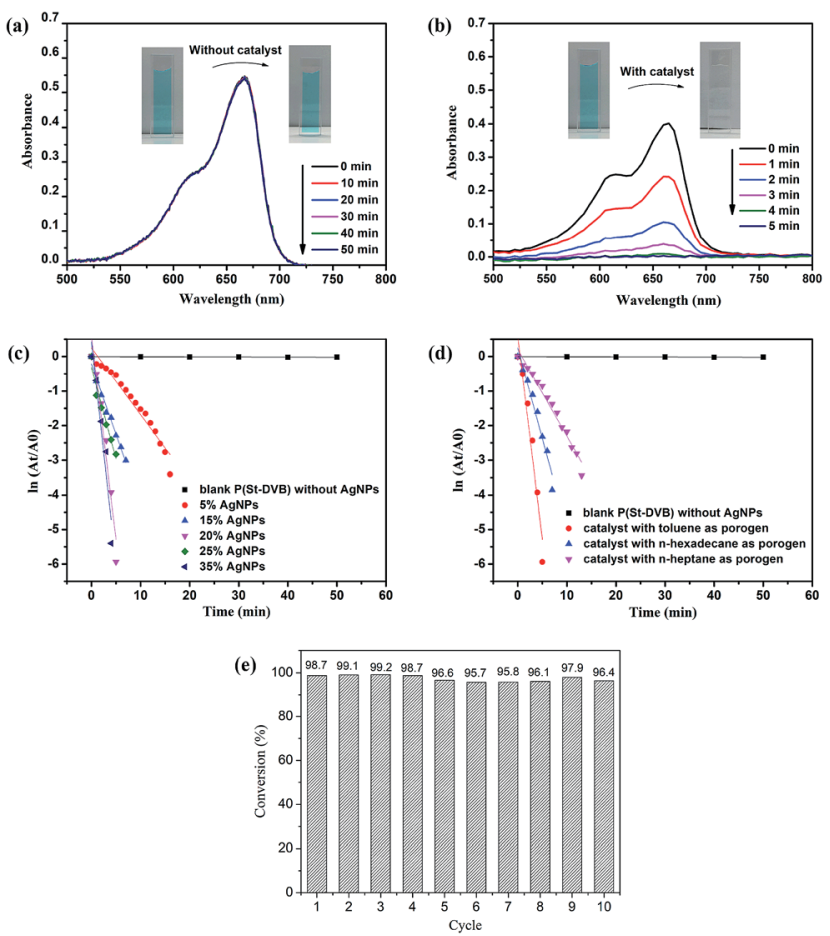

Fig. 13 Catalytic performance of the porous $P(S t-D V B) / A g N P s$ composite microspheres: UV-Vis absorption spectra of MB solution in the presence of $\mathrm{NaBH}_{4}$ with (a) blank porous $\mathrm{P}(\mathrm{St}-\mathrm{DVB}$ ) and with (b) the porous $\mathrm{P}(\mathrm{St}-\mathrm{DVB}) / \mathrm{AgNPs}$ composite microspheres, and the insets were corresponding photographs of the reaction solution in the presence of $\mathrm{NaBH}_{4}$ before and after the catalytic reactions; (c) the plots of $\ln \left(A_{t} / A_{0}\right)$ versus reaction time for catalytic reduction of $M B$ with different loading content of AgNPs in porous P(St-DVB)/AgNPs composite microspheres; $(d)$ the plots of $\ln \left(A_{t} / A_{0}\right)$ versus reaction time for catalytic reduction of MB with different porogen used porous $\mathrm{P}(\mathrm{St}$ DVB)/AgNPs composite microspheres; (e) conversion of MB in 10 successive cycles with the composite microspheres.

nitroanilines and various dyes, such as methylene blue (MB) and methyl orange. ${ }^{52,53}$ Herein, the catalytic degradation property of $\mathrm{P}(\mathrm{St}-\mathrm{DVB}) / \mathrm{AgNPs}$ composite microspheres for organic dyes was investigated. MB was selected as a model dye and its evolution of UV-Visible spectra at the wavelength of absorbance maximum $\left(\lambda_{\max }\right)$ at $665 \mathrm{~nm}$ during the reduction was illustrated in Fig. 13(a and b). Without catalyst addition, the peak intensity at $665 \mathrm{~nm}$ did not have a significant change in 50 min even in the presence of $\mathrm{NaBH}_{4}$ in Fig. 13(a). By contrast, after the addition of the $\mathrm{P}(\mathrm{St}-\mathrm{DVB}) / \mathrm{AgNPs}$ composite microspheres, the intensity of the absorbance peak at $665 \mathrm{~nm}$ declined significantly with time in Fig. 13(b). The occurrence of rapid degradation with the addition of the composite microsphere catalyst was further confirmed by comparing the corresponding color change of the solutions (insets of Fig. 13(a and b)). The blue solution faded into colorless with the addition of the composite microsphere catalyst in $5 \mathrm{~min}$, while the color of the solution without the catalyst remained blue.

In this research, the concentration of $\mathrm{NaBH}_{4}$ was chosen to largely exceed the concentration of MB. Thus the kinetics of the reduction could be treated as pseudo-first order for the $\mathrm{MB}$ 
concentration. The rate constant of this reaction is calculated using the formula (1).

$$
\ln \left(A_{t} / A_{0}\right)=-k_{\text {app }} t
$$

where $k_{\text {app }}$ is the apparent rate constant, $t$ is the reaction time, $A_{0}$ is initial concentration of $\mathrm{MB}$, and $A_{t}$ is concentration of $\mathrm{MB}$ at time $t$. In Fig. 13(c and d), the influences of AgNPs loading contents on the composite microsphere catalysts and the porous structures of the composite microsphere catalysts on the catalytic activity were investigated. To investigate the influence of AgNPs loading contents on the catalytic activity, samples S1, S2, S6, S11 and S12 were used, which were listed in Table 3. The samples obtained from different porogens listed in Table 4 (S6, S13 and S14) were used to investigate the influence of porous structures of the composite microspheres on the catalytic activity. In order to eliminate differences of the AgNPs loading contents from different structured samples, $10 \mathrm{mg}$ S6 (toluene as porogen), $10.87 \mathrm{mg} \mathrm{S} 13$ ( $n$-hexadecane as porogen) and $13.36 \mathrm{mg}$ S14 (n-heptane as porogen) catalysts were added respectively in the catalytic reaction, which is calculated from the data of TGA curves in Fig. 12.

In summary, from the results of Fig. 13(c), the catalytic rate increased with increasing content of the AgNPs loaded on the microspheres, i.e. the AgNPs played the most important role on the catalysis. In Fig. 13(d), the rate constants $k_{\text {app }}$ of composite microspheres with toluene, $n$-hexadecane and $n$-heptane as porogens were $1.17 \mathrm{~min}^{-1}, 0.52 \mathrm{~min}^{-1}$ and $0.25 \mathrm{~min}^{-1}$ respectively, which means the mesoporous structure came from toluene as porogens benefits the catalytic degradation of MB.

In addition to the catalytic activity, reusability is critically important for practical dye degradation applications. The repeated catalytic performance of the $\mathrm{P}(\mathrm{St}-\mathrm{DVB}) / \mathrm{AgNPs}$ composite microspheres were shown in Fig. 13(e). The composite microsphere catalyst was still able to degrade $\sim 96 \%$ of $\mathrm{MB}$ at the $10^{\text {th }}$ run, indicating excellent recyclability of the porous $\mathrm{P}(\mathrm{St}-\mathrm{DVB}) / \mathrm{AgNPs}$ composite microspheres as a catalyst. The excellent recyclability was attributed to the affinity of AgNPs to the porous $\mathrm{P}(\mathrm{St}-\mathrm{DVB})$ substrates.

\section{Conclusions}

In this research, porous $\mathrm{P}(\mathrm{St}-\mathrm{DVB}) / \mathrm{AgNPs}$ composite microspheres with tunable porosity were prepared through a one-pot preparation method based on seed swelling polymerization by directly dispersing self-synthesized organic-soluble AgNPs into the mixture of reactive chemicals. The tunable porosity was obtained mainly by regulating the ratio between the monomer styrene and the crosslinker DVB, by adjusting the amount of AgNPs adding into the polymerization and by changing the type of porogens used during the polymerization. The prepared porous $\mathrm{P}(\mathrm{St}-\mathrm{DVB}) / \mathrm{AgNPs}$ composite microspheres showed excellent catalytic activity in the degradation of methylene blue with $\mathrm{NaBH}_{4}$. The catalytic rate was influenced by the amount of AgNPs loaded on the composite microspheres as well as the pore type of the composite microspheres. The degradation results showed mesopores obtained by using toluene as porogen and had the highest catalytic rate compared to the macropores and micropores structures. The excellent catalytic performance of $16.7 \%$ AgNPs loaded mesopores P(St-DVB)/ AgNPs composite microspheres was demonstrated after 10 catalytic cycles. Thus a good stability and durability of AgNPs was obtained by using mesopores P(St-DVB) as a matrix. In the future, this kind of novel composite materials may have great potential in the application for water and gas treatment, energy storage, antibacterial agent and SERS sensing.

\section{Conflicts of interest}

There are no conflicts to declare.

\section{Acknowledgements}

This work was supported by Shanghai academic and technological leaders program (16XD1424500). The author X. Ji gratefully acknowledges the Donghua University (DHU) Special Excellent PhD International Visit Program partial supports of this work and financial support from Prof. Dr H.-U. Moritz.

\section{Notes and references}

1 B. Sun, B. Hua, X. Ji, Y. Shi, Z. Zhou, Q. Wang and M. Zhu, Mater. Res. Innovations, 2014, 18, 869-874.

2 S. Chernousova and M. Epple, Angew. Chem., Int. Ed., 2013, 52, 1636-1653.

3 C. Ma, M. J. Trujillo and J. P. Camden, ACS Appl. Mater. Interfaces, 2016, 8, 23978-23984.

4 C. Gao, Z. Lu, Y. Liu, Q. Zhang, M. Chi, Q. Cheng and Y. Yin, Angew. Chem., Int. Ed., 2012, 51, 5629-5633.

5 C. Jiang and F. Schüth, Phys. Chem. Chem. Phys., 2011, 13, 2457-2487.

6 Y. Wen, H. Ding and Y. Shan, Nanoscale, 2011, 3, 4411-4417.

7 B. Baruah, G. J. Gabriel, M. J. Akbashev and M. E. Booher, Langmuir, 2013, 29, 4225-4234.

8 Y. Shiraishi and N. Toshima, J. Mol. Catal. A: Chem., 1999, 141, 187-192.

9 D. B. Ingram and S. Linic, J. Am. Chem. Soc., 2011, 133, 52025205.

10 Z. Jiang, C. Liu and L. Sun, J. Phys. Chem. B, 2005, 109, 17301735.

11 G. Yang, G. Gao, C. Wang, C. Xu and H. Li, Carbon, 2008, 46, 747-752.

12 H. Liu, J. Bai, S. Wang, C. Li, L. Guo, H. Liang, T. Xu, W. Sun and $\mathrm{H}$. Li, Colloids Surf., A, 2014, 448, 154-159.

13 Y. Li, Y. Cao, J. Xie, D. Jia, H. Qin and Z. Liang, Catal. Commun., 2015, 58, 21-25.

14 N. Salam, B. Banerjee, A. S. Roy, P. Mondal, S. Roy, A. Bhaumik and S. M. Islam, Appl. Catal., A, 2014, 477, 184-194.

15 W. Zhang, Y. Sun and L. Zhang, Ind. Eng. Chem. Res., 2015, 54, 6480-6488.

16 G. Li and Z. Tang, Nanoscale, 2014, 6, 3995-4011.

17 M. M. Mohamed and M. S. Al-Sharif, Appl. Catal., B, 2013, 142-143, 432-441. 
18 D. Li, M. Dong, F. Besenbacher, Y. Huang and M. Chen, RSC Adv., 2016, 6, 65613-65618.

19 J. Fan, C. Huang, L. Jiang and S. Wang, J. Mater. Chem. B, 2013, 1, 2222-2235.

20 D. Yuan, W. Lu, D. Zhao and H. Zhou, Adv. Mater., 2011, 23, 3723-3725.

21 A. S. P. Lin, T. H. Barrows, S. H. Cartmell and R. E. Guldberg, Biomaterials, 2003, 24, 481-489.

22 U. Mandi, A. S. Roy, S. K. Kundu, S. Roy, A. Bhaumik and S. M. Islam, J. Colloid Interface Sci., 2016, 472, 202-209.

23 J.-W. Kim, J.-E. Lee, J.-H. Ryu, J.-S. Lee, S.-J. Kim, S.-H. Han, I.-S. Chang, H.-H. Kang and K.-D. Suh, J. Polym. Sci., Part A: Polym. Chem., 2004, 42, 2551-2557.

24 T. Zha, L. Song, P. Chen, W. Nie and Y. Zhou, Colloids Surf., A, 2015, 481, 423-430.

25 M. T. Gokmen and F. E. Du Prez, Prog. Polym. Sci., 2012, 37, 365-405.

26 T. D. Dao, G. Erdenedelger and H. M. Jeong, Polymer, 2014, 55, 4709-4719.

27 G. Li, H. Möhwald and D. G. Shchukin, Chem. Soc. Rev., 2013, 42, 3628-3646.

28 N. Khalid, I. Kobazashi, M. A. Neves, K. Uemura, M. Nakajima and H. Nabetani, Colloids Surf., A, 2014, 458, 69-77.

29 B. Kim, T. Y. Jeon, Y. K. Oh and S. H. Kim, Langmuir, 2015, 31, 6027-6034.

30 M. Albuszis, P. J. Roth, F. Exnowitz, D. L. Wong, W. Pauer and H.-U. Moritz, Polym. Chem., 2016, 7, 1168-1180.

31 Q. Tian, D. Yu, K. Zhu, G. Hu, L. Zhang and Y. Liu, J. Colloid Interface Sci., 2016, 473, 44-51.

32 Y. Yang, H. Liao, Z. Tong and C. Wang, Compos. Sci. Technol., 2015, 107, 137-144.

33 Q. Tian, X. Yu, L. Zhang and D. Yu, J. Colloid Interface Sci., 2017, 491, 294-304.

34 Y. Shi, B. Sun, Z. Zhou, Y. Wu and M. Zhu, Prog. Nat. Sci.: Mater. Int., 2011, 21, 447-454.

35 M. Albuszis, P. J. Roth, W. Pauer and H.-U. Moritz, Polym. Chem., 2014, 5, 5689-5699.
36 V. S. Suvith and D. Philip, Spectrochim. Acta, Part A, 2014, 118, 526-532.

37 M. Okubo, M. Shiozaki, M. Tsujihiro and Y. Tsukuda, Colloid Polym. Sci., 1991, 269, 222-226.

38 K. Lewandowski, F. Svec and J. M. J. Fréchet, Chem. Mater., 1998, 10, 385-391.

39 H. Kobayashi, E. Miyanaga and M. Okubo, Langmuir, 2007, 23, 8703-8708.

40 X. H. Zhao, Q. Li, X. M. Ma, Z. Xiong, F. Y. Quan and Y. Z. Xia, RSC Adv., 2015, 5, 49534-49540.

41 J. Zhang, X. Ge, M. Wang, J. Yang, Q. Wu, M. Wu and D. Xu, Polym. Chem., 2011, 2, 970-974.

42 D. M. Dotzauer, J. Dai, L. Sun and M. L. Bruening, Nano Lett., 2006, 6, 2268-2272.

43 M. Galia, F. Svec and J. M. J. Fréchet, J. Polym. Sci., Part A: Polym. Chem., 1994, 32, 2169-2175.

44 C. Viklund, F. Svec and J. M. J. Fréchet, Chem. Mater., 1996, 8, 744-750.

45 Y. Hu, T. Zhao, P. Zhu, X. Liang, R. Sun and C. Wong, RSC Adv., 2015, 5, 58-67.

46 F. S. Macintyre and D. C. Sherrington, Macromolecules, 2004, 37, 7628-7636.

47 Y. Konishi, M. Okubo and H. Minami, Colloid Polym. Sci., 2003, 281, 123-129.

48 M. J. Bene, D. Horák and F. Svec, J. Sep. Sci., 2005, 28, 18551875.

49 M. Okubo, Y. Konishi and H. Minami, Prog. Colloid Polym. Sci., 2004, 124, 54-59.

50 S. Dubinsky, J. Park II, I. Gourevich, C. Chan, M. Deetz and E. Kumacheva, Macromolecules, 2009, 42, 1990-1994.

51 M. Thommes, K. Kaneko, A. V. Neimark, J. P. Olivier, F. Rodriguez-Reinoso, J. Rouquerol and K. S. W. Sing, Pure Appl. Chem., 2015, 87, 1051-1069.

52 N. Gupta, H. P. Singh and R. K. Sharma, J. Mol. Catal. A: Chem., 2011, 335, 248-252.

53 X. Liu, H. Cheng and P. Cui, Appl. Surf. Sci., 2014, 292, 695701. 